\title{
Collocated Interaction with Flying Robots
}

\author{
Wai Shan (Florence) Ng, Ehud Sharlin
}

\begin{abstract}
We introduce a socially motivated interaction technique with collocated flying robots (a quadrotor in our current prototype). Instead of the traditional remote interaction controllers often used when interacting with flying robots and UAVs, we explore the collocated interaction space and suggest a direct interaction technique motivated by social human-robot interaction themes. Our approach is inspired by the types of interaction humans have with birds, specifically falconeering, and is facilitated by gestures-based interaction, while the user is within the field of view of the flying robot. This paper outlines our research goals, task examples, and our overall design approach. The paper also discusses our current prototyping efforts, as well as a preliminary evaluation of our approach, performed through two design critiques, studying our collocated interaction technique concept, and its potential, drawbacks and benefits for users.
\end{abstract}

Keywords-social human-robot interaction, flying robot, $U A V$, gesture-based interaction, collocated interaction, Wizard of $\mathrm{Oz}$ Evaluation

\section{INTRODUCTION}

$\mathrm{C}$ IONTROLLING unmanned aerial vehicles (UAVs, or drones) is a task that is both daunting and necessary. UAVs are used extensively in the defense and security domains and are making their way into the general market in various forms, notably that of quadrotor toys. Currently, controllers and pre-planned flight path programs are the norm for controlling drones and much research has been done in effort to make controlling UAVs as simple and as intuitive as possible [12]. Remote interaction is generally the only valid approach when the flying robot is far away from the user. However, we believe that when the robot is close to the user it may be advantageous to move away from the current paradigm of remotely controlling the robot and instead pursue socially inspired direct interaction metaphors, based on modalities such as body gestures and voice. When humans are in close proximity with others they wish to interact with, they would simply face them and gesture or talk to them directly. It would be awkward for both parties to start a webcam session with one another when they are in the same room. Similarly, we believe it would be more natural to interact with a flying robot which is hovering next to the user without using a screen and joystick, but rather with a set of direct interaction metaphors, such as body gestures.

When a flying robot is far away from the user, a social disconnection is inevitable. However, when communicating

Wai Shan (Florence) $\mathrm{Ng}$ completed her BSc. in computer science at the University of Calgary and is currently continuing her research at the Interactions Lab at the university. (e-mail: wsng@ucalgary.ca)

Ehud Sharlin is currently an Associate Professor at the University of Calgary. (e-mail: ehud@ucalgary.ca)

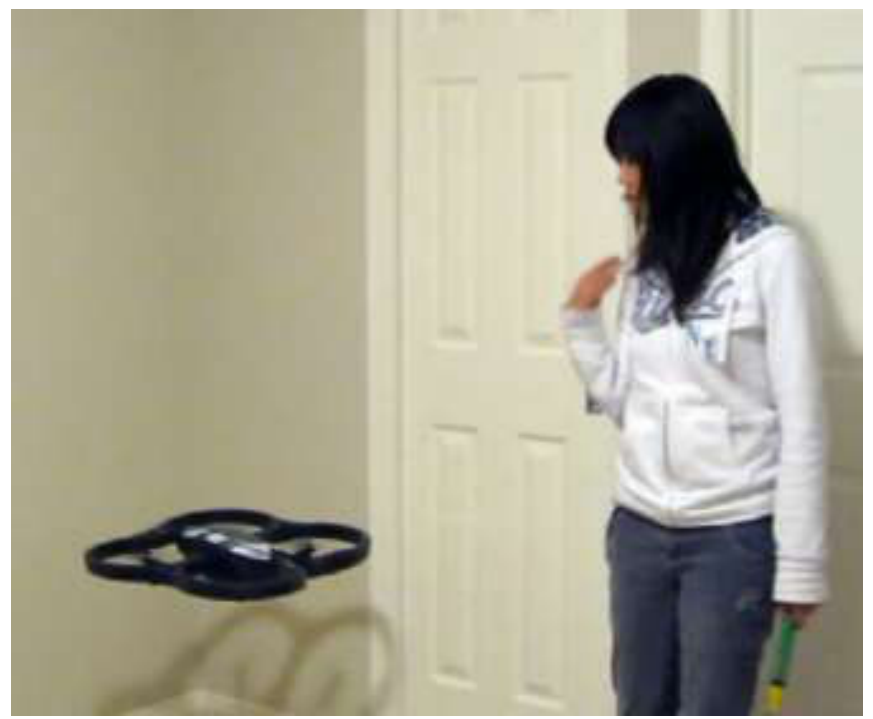

Figure 1. Using a gesture to indicate to a robotic drone to fly closer.

with a flying robot within the same locality we believe that the natural instinct is to engage with the robot using either speech or body gestures, similar to how people engage with other collocated people or animals.

Currently drones are mostly used for remote surveillance and security tasks which occur remotely, away from the user. Following, drones do not fly in the user's collocated space and there is no advantage of using body gestures rather than a controller. However, we believe that in time, flying robots will become entities that can be collocated with users, and eventually even play a role as a social collaborator for various tasks. For inspiration we look at the relationships humans have with birds. For centuries, falconers and their birds have collaborated in capturing prey together. They use arm movement, body posture and bait to tell the bird of prey what it needs to do. Of course, there are major differences between a drone and a falcon, and current drones can be controlled and told to perform more tasks than a falcon. However, the similarities can be interesting to explore. We envision that in the future flying robots may stay close to their users as a companion, and wait for tasks. It is from the falconers' interaction with their birds that we draw inspiration for tasks and commands that users may wish to use when interacting with a collocated flying robot. We see our current efforts as only a first step towards much richer collocated interaction between humans and flying robots.

Although there is considerable research on social interaction with robots, interfaces for flying robots have so far, to the best of our knowledge, ignored the social, collocated aspect. Taking inspiration from the interaction between 
falconers and their birds of prey, as well as from common gestures in our day to day lives, we propose a gesture-based interaction scheme that attempts to create a more intuitive and natural way to communicate with flying robots. Our approach is based on a collocated space (outdoors or indoors) which the robotic drone and the user share, and on a set of simple physical gestures that the user employs to interact with the robot as long as the drone is maintaining a line-of-sight with the user.

In this paper, we discuss our design approach for a gesture based interface with collocated flying robots, the current early prototype we designed, as well as a set of preliminary design critiques. We first asked the participants to critique the gestures we suggest without the drone present, in order to evaluate the validity of the proposed interaction. In a second design critique, we asked the participants to interact with a flying robot using gestures using the Wizard of $\mathrm{Oz}$ (WoZ) approach to explore the benefits and drawback of our collocated social approach to interaction with flying robots. Our design critiques were exploratory and based on a small number of participants who were generally aware of the nature of the study. This preliminary evaluation is not meant to replace an extensive study, but to provide insight regarding the potential of the gesture based interaction approach with flying robots, and to help us gather requirements for the next phases of the system development.

\section{RELATED WORK}

Quigley et al. [11] explored several interfaces to control UAVs, ranging from a traditional numerical input interface where the user must input numerical values when controlling the flight of a drone, to using a physical tangible model of the plane as the controller. Quigley et al. used a PDA to control the non-physical interaction schemes, and focused on controlling a remote drone rather than on the collocated interactions. However, of particular interest to us in Quigley et al.'s effort is the voice control interaction. In this part of their study the user talked to the PDA in order to control the remote UAV. We do not plan on using voice control at this point, but we see this interaction modality as very relevant to collocated interaction with flying robots, and thus as closely related to our research effort.

There are many examples of exploring gestures based interaction with collocated ground robots (e.g. [2, 13]). For example, Iba et al. [9] uses hand gestures to control both the low-level and high-level movements of a robot. Their system also includes a teleportation mode which allows the user to use gestures to control the robot when it cannot see the user. As far as we are aware, we are the first to control a flying robot using gestures in collocated space. While the gestures component of our effort is quite similar to those of collocated interfaces with ground robots, we believe that a flying robot has interesting factors that need to be consider compared to a robot that travels by land (e.g. more spatial flexibility, pitch and roll) and that highlights the uniqueness and challenges of designing a collocated gesture based interaction with a flying robot.

The presence of a physical robot in the user's collocated space affects the way the person interacts with it, as seen in the research done by Bainbridge et al. [2]. The participants were more inclined to trust a robot that was physically present than a virtual robot, and could interpret the robot's actions better. Our flying robot interacts with the user by following the gestures rather than a pre-programmed task list, and is very much present in the user's environment. Based on this we also expect to see the emergence of stronger social relationship and emotions such as trust (or fear) than those that would be expected when interacting with a remote robot.

\section{DESIGN APPROACH AND PRELIMINARY PROTOTYPE}

In order to examine the high level research vision of collocated interaction with flying robots, we thought of designing a minimal interface that would allow us to gain insight on whether this approach makes sense to users.

Our focus for the first phase of the research was the interaction metaphor and its validity, not the implementation of the interface. Although we explored specific gestures to be used with the flying robot, it is not the gestures that are most interesting to us, but learning how the users react to the interaction, and trying to understand the strength and weaknesses of our approach.

For our preliminary prototyping efforts we decided to employ a quadrotor, a flying robot with four rotating blades which can take off and land vertically like a helicopter. Quadrotors can be lightweight and small, and may be able to carry a small load depending on the model.

We are currently using Parrot's A.R. Drone in our design (Figure 1) as it is geared towards the consumer market, affordable and still quite capable to fulfill our short term research goals. The A.R. Drone is equipped with an accelerometer and two gyrometers, as well as a front facing camera and a bottom camera. The A.R. Drone uses an Apple iPod touch/iPhone and an app that allows the user to control it with buttons and the tilt of the device.

We decided to take a WoZ approach [5] to evaluate the gestures-based interactions between the user and the collocated drone. With this approach, although the participants interact with the robot directly with gestures and see it perform the corresponding task, the robot is actually controlled by an experimenter (the "wizard") who is closely following the participants' gestures and commands the drone according to the interface algorithm or state machine. The WoZ technique allowed us to gain insight on whether our approach has potential validity for users, and to examine in practice some of the tasks and interaction techniques we envision before committing to longer and more demanding implementation. The obvious limitations of this approach is that it is currently not tackling the technical challenges of recognizing the gestures and autonomously controlling the robot, and is thus 

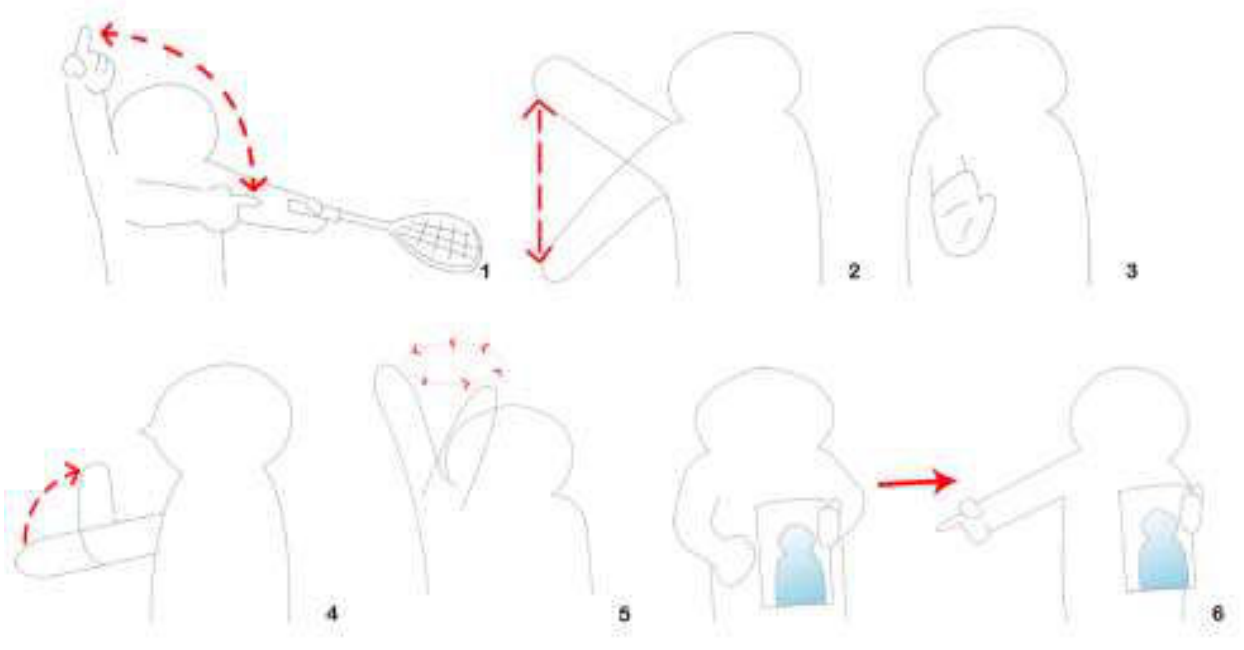

Figure 2. An illustration showing the prototype of our interaction gestures with a collocated flying robot. From 1 to 6: takeoff and land; raise and lower; stop; come; circle; and find.

still far from deployment in a study that does not involve a "Wizard" operator. Although we decided to use WoZ without deceiving the users (see for example [8]), we believe the results are still valid as they provide preliminary insight into the strengths and weaknesses of our approach, and the validity of the mapping between gestures and tasks.

\section{TASKS AND GESTURES}

Although robotic appliances and companions are still rare at the moment, we believe that robots will work much closer with the general public in the near future. Robots that travel by land are already becoming more prevalent among the general public, in tasks such as vacuuming and lawn-mowing. However, robots that are limited to moving along the ground will not be able to reach specific locations or viewpoints that can be more accessible to a flying robot.

A flying robot might be sent to check the traffic conditions ahead of a user's vehicle while stuck in a traffic jam, or to retrieve a tool from the top floor of a skyscraper. Since a flying robot does not depend on the ground conditions, it can reach these areas much quicker than a land-bound robot. A flying robot can provide the user with a wide overview of an area, quickly. It may be able to locate entities, objects or people, given this wide view point, very much like a hawk helps its falconer find a rabbit. A flying robot can also become a pet, similar to a parrot. We believe that as drones become smaller and smarter the concept of a small flying robot becoming a collocated social entity, and perhaps even a collaborator and companion, will become a reality.

Controlling a flying robot effectively can prove difficult for untrained users [6]. The tasks we suggest are high level and based on an underlying layer of automation: for example, a task would enable the user to gesture to the robot to circle over her head, but will not depend on the user's awareness and ability to control the pitch and roll of the robot in order to actually facilitate the circling act.

Although we have considered using Semaphore flag signaling [4], a Landing Signal Officer (LSO)'s signals [10], and sign language as a basis for the gestures, there are different problems associated with each.

The Semaphore flag system uses two flags, and the position of the flag indicates a letter in the English alphabet. Using a series of flag positions, a person can spell a message to another person. This is much too complicated to memorize for an untrained user, and the gestures would not be a natural mapping to the quadrotor's tasks.

The LSO's signals are designed specifically for landing an aircraft. Therefore, the meaning of the signals are task specific and thus limited, and do not cover a wide range of actions. The actions are low level and focus on the mechanics of the plane rather than the higher level tasks we are concerned with. If we were to use the LSO gestures vocabulary, but change the meaning of the gestures, then it would result in a set of gestures which is arguably not much better than the Semaphore flag system in terms of the lack of natural mapping to the tasks.

Using sign language can be a good match, as it has a large vocabulary with many actions that can be expressed using only a small set of hand gestures. On the other hand, one gesture may have multiple meanings, so the gesture may not map effectively to our tasks which are specifically targeted for flying robots. The stop sign for example can be described as hitting the side of the hand on the palm of the other hand. Though this makes sense in some context, it may not be so clear to users who are not familiar with sign language that the gesture tells the quadrotor to stop and hover in space. We believe that most people are more familiar with the proposed gesture for stopping as it is a gesture traffic police officer often use when directing traffic. 


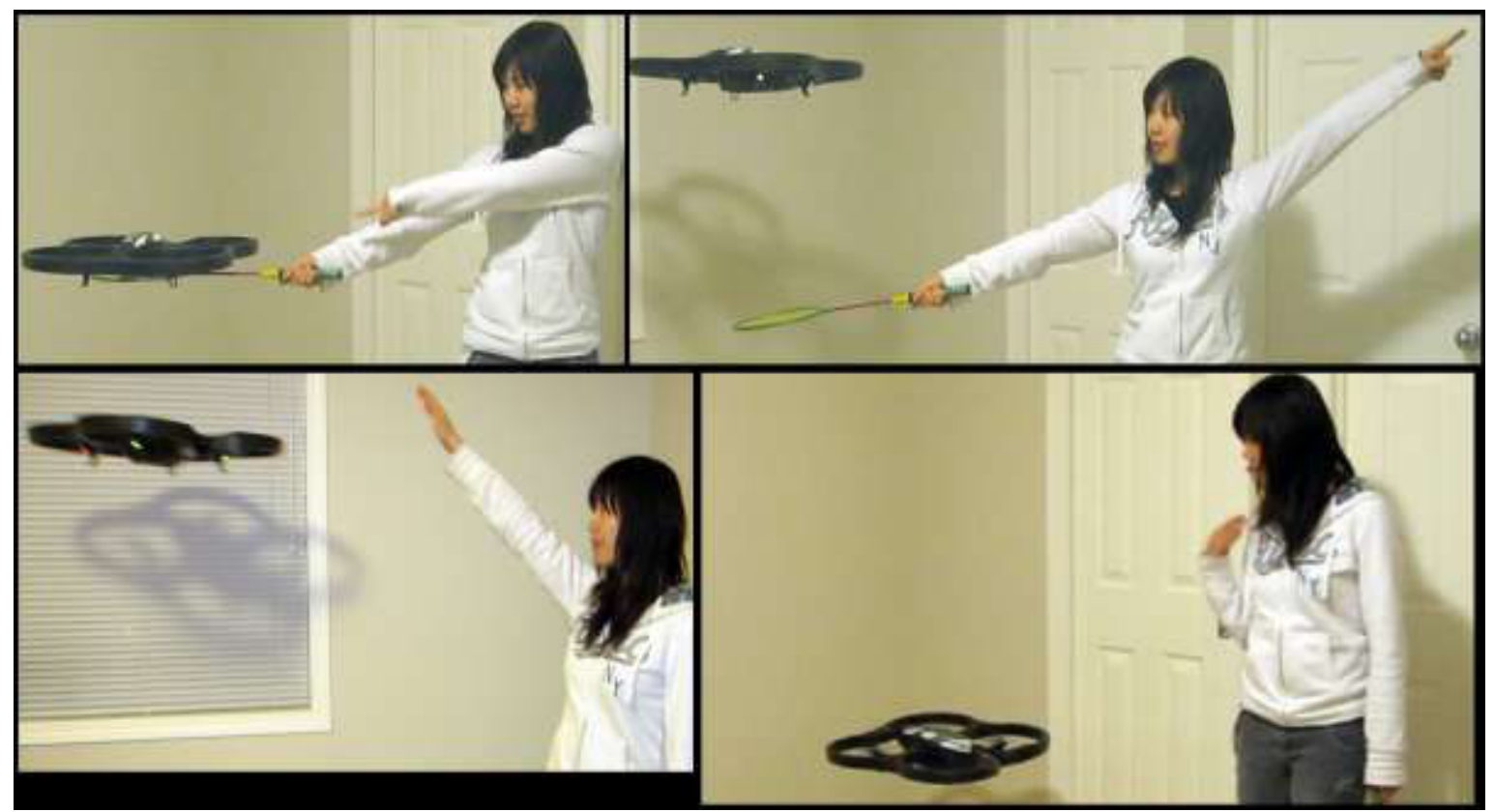

Figure 3. (Top) A user doing the takeoff gesture with a badminton racquet as the takeoff pad. (Bottom left) Making a raise gesture. (Bottom right) Making a come gesture.

In the following section, we outline a small set of basic tasks we selected to examine the validity of collocated gesturebased interaction with a flying robot, and to suggest a set of specific gestures that would allow the user to communicate these interactive tasks to the collocated flying robot. Ours is by far not a comprehensive list of tasks that can be done with a collocated flying robot, and are used only in order to illustrate and later evaluate the general approach we are proposing. These gestures (see Figure 2) are only meant to be used when the quadrotor is close to the user and when a line-of-sight can be maintained.

Because of the A.R. Drone's rotating blades and the risk of scaring (or harming) the user if the quadrotor is too close to the user's body we decided to use a handheld takeoff and landing pad (Figure 3). The pad allows the user to hold the drone away from the body, and provide a flat stable surface for the drone to land on, or takeoff from. Other than the takeoff and landing gestures, the flying robot is at a far enough distance away from the user, making the pad unnecessary for safety concerns. The user may choose to keep holding the pad for convenience however. With a different type of flying robot (for example, one with a propeller that is less exposed, or much weaker), it may be possible to forgo the pad altogether.

The following section outlines six tasks and the corresponding gestures: takeoff and land, raise and lower, stop, come, circle, and find.

\section{A. Takeoff and Land}

Takeoff is a very important task for operating a drone. When falconers wish to send a bird out to catch a prey or for training purposes they will gently move their hand, almost tossing the bird into its intended flight direction, while it is on the falconer's arm. Our design of the takeoff gesture is inspired by this falconeering gesture. In the takeoff gesture the user holds the pad with the drone on it at arm's length (Figure 3). To make the gesture, the user holds the pad with the drone on it perpendicular to her body, and points at the drone, arm straight, with the other hand. Then the user moves the arm up in an arcing motion until she is pointing straight up.

Like taking off, landing is a major task when operating a quadrotor. Again, our gesture design was inspired by the falconer holding an outstretched arm for the bird to land on. In our designed gesture, the user outstretches one arm to hold the pad for the drone to land on and uses a gesture with the other arm similar to the takeoff motion: pointing at the drone, and then moves the arm to the pad.

\section{B. Raise and Lower}

Adjusting the flying altitude can be done by lifting or lowering an extended, straight arm. If the arm is held at the maximum height of the raising motion, the drone will keep raising until the user retracts her arm, or until the drone cannot fly higher. The same holds for the lowering gesture, lowering the arm will lower the drone, till ground level is reached, or the arm is retracted (Figure 3).

\section{Hover}

We prototyped three types of hovering: maintaining a stationary position in one location (stop); following the user (come); and circling around the user (circle).

To gesture to the drone to hover in one spot, the user should hold out the palm of her hand to the drone. The drone will then hover at its current location and altitude. To tell the drone to follow the user, she should hold out her arm straight perpendicular to the body, then bend the elbow up close to the body to make a "come" motion (Figure 3). The quadrotor will follow beside the user. Finally, to have the drone circle around the user, the user simply traces a circle with her arm around 
her head. The radius of the circle dictates the relative radius of the drone's circular flight path.

\section{Find}

This task is considered a high level task, since it concerns an abstract concept, beyond spatial control, and involves several steps. There are several ways to introduce the drone with the person (or perhaps in a slightly different variant, an object) the user wants to find, such as showing a picture of the person to the quadrotor, or if the target object is tagged, for example with a Quick Response (QR) code marker, the user can introduce the code to the drone, rather than a picture. Once the image or code is introduced the user holds up a fist near it, to indicate to the robot that it should search for the person or object that was introduced to it via the picture or the marker. The user can then point to a direction she wants the drone to search initially. A time limit should be implemented, telling the drone to fly back to the user in case it failed to find the person or the object.

\section{FIRST DESIGN CRITIQUE}

In order to provide a preliminary design critique of our gestures approach to interaction with a flying robot we recruited two senior computer science students as expert users, and one non-computer science student as a non-expert participant. In this preliminary design critique we focused on the validity of the gestures only, and refrained from flying the quadrotor next to the participant. The gestures were first demonstrated and explained to the participant, then we asked $\mathrm{him} / \mathrm{her}$ to perform the gestures while imagining a quadrotor flying above them. Our goal was to find out what the participants thought of the gestures, tasks, and the idea of interacting with a flying robot using gestures before attempting to control a robot to perform the tasks based on the gestures. One of the participants was given a badminton racquet as a pad for the takeoff and land gestures.

\section{A. Results}

The participants generally found the tasks were easy to understand, and commented that they believe these can be useful for operating the quadrotor in most situations. All of the participants liked the idea of using gestures to control the flying robot. The participants liked the stop and come gestures the most, as these are common gestures the participants use and see often.

One participant suggested the stop task should be the normal state of the quadrotor and thus unnecessary to make a gesture for it.

Another participant did not like the raising and lowering motion as the arm was diagonal to the body. He argued that the action suggests the quadrotor will move diagonally rather than vertically, as actually intended. He also noted that there was no real need to make the raising action, and that perhaps a stationary action would suffice. He suggested a thumbs-up or thumbs-down action as an alternative. Another participant expressed that the original raising gesture was fine as long as the task was explained to the user.

The participants' opinion on the circling hover motion was somewhat split as well. One thought the motion was too excessive, and can be limited to a circle in front of the user, using just the forearm for the circling movement. Another participant commented that the motion was excessive and may be embarrassing to do in public. The other participant argued that using the entire arm may be necessary for the full range of different radii a user may want the quadrotor to circle.

One of the participants wanted to be able to combine the gestures by using both arms. His example was a task where the quadrotor should circle, not around the user, but at another location. The gesture should then combine the circling motion with a pointing motion.

When searching for an object or a friend, one participant suggested making a radius motion to specify a range that the quadrotor should look within. He suggested that a directional cue can be incorporated into the gesture by making the arm gesture to the side of the body in which the user wants the quadrotor to go. The non-expert participant was confused with the fist gesture, and commented that it did not map to the intended task. She felt the gesture may indicate that the quadrotor should attack the person or the object in the picture. She suggested that using the fingers as a frame around the picture may be a better, more valid gesture.

The participant using the racquet raised a concern that the user may become fatigued if holding out the racquet for an extended amount of time, especially if the drone is located on top of it. She also pointed out that needing to bring the racquet or a similar pad whenever a user wants to use the quadrotor may be quite inconvenient.

\section{B. Discussion}

Based on the preliminary design critique we examined the gestures and considered revising them. We decided to keep the stop motion, and but modify it so that it also became a way of cancelling the quadrotor's current task, such as circling.

There is merit in both views on the amount of motion needed to make the circle gesture, and based on the results we are uncertain which is more effective. New separated gestures can be used to tell the flying robot to fly in a larger or smaller circular path.

In the case of wanting the quadrotor circling at a different location, we believe it is a problem with the task definition, rather than a case where one would need to combine two actions. Following, we decided that the circling task would be modified so the quadrotor will fly in a circular path with its current location as the center of the circle. If the user then wants to have the quadrotor circle around her as before, she can make the come gesture after telling the quadrotor to circle.

Although there may be some situations such as the above when it would be advantageous to combine two gestures, it may be hard for the user to determine what the quadrotor will do if the two gestures conflict. If the need arises for two 
gestures to be combined to make a natural and easily understood movement, the combination of the gestures can simply be defined as a new gesture. This may lead to a system with many gestures, which may end up being confusing for users. Thus we decided not to consider using multiple gestures for the next design critique.

The size of the badminton racquet we used, and the inconvenience of carrying it around is a valid problem, but the size of the badminton racquet is very close to the size of the A.R. Drone itself which makes carrying together or separately, almost as cumbersome. The weight of the racquet combined with the quadrotor is approximately $550 \mathrm{~g}$ which, when held outstretched may indeed cause fatigue especially in the arm.

Overall, the comments were favorable in support of using gestures for communicating with a flying robot. Some gestures - raising/lowering, circling, finding a friend - needed some adjustments, before we tested them in the second design critique.

\section{SECOND DESIGN CRITIQUE}

For this design critique we recruited one male computer science expert, and an 11 year-old boy who has no experience in the domain, or in controlling flying toys. In this design critique the participants were asked to control the quadrotor using the initial set of gestures as well as the revised gestures that resulted from the first design critique. The evaluation was based on an informed WoZ evaluation technique, with no deception. Though both participants knew that the experiment administrator was controlling the quadrotor from behind the scenes, they were asked to pretend that the quadrotor moved autonomously, following the gestures given. The second design critique was thus unique as it explored the gestures, but integrated the flying robot's collocated presence and movement as an important factor impacting the interaction. Manually controlling the drone based on the gestures was often difficult, so at times the quadrotor would crash or fly in the wrong direction.

\section{A. Results}

Though the adult participant was not afraid of the quadrotor, the child was quite nervous when the quadrotor came close by. When taking off, even with the racquet extended the younger participant was afraid of the quadrotor. However, after showing him that he can push it away, he became more confident around it.

When the drone crashed or flew in an unexpected manner, the adult participant was reminded that the drone was controlled by the experimenter, but the child acted as if the quadrotor was malfunctioning, and avoided it until it stabilized.

The child was very engrossed in the interactions with the quadrotor, and talked to it while doing the gestures as well. He congratulated the drone when the right action was done correctly, and would say "no, no" when the quadrotor does not do what it was told. When the quadrotor crashed, he asked with concern, "is it okay?"

He also enthusiastically performed the gestures, and sometimes over-exaggerated them. For the lowering gesture, he not only lowered his arms, but also bended his legs so his hands was even closer to the ground. He was under the perception that if he used both arms for the gestures, the quadrotor would perform the action faster, thus he used both arms for the raise, lower, and come gestures.

After becoming fully immersed in the critique, the younger participant modified the raising and lowering motion from the diagonal arm up/down to moving the arm vertically up/down. The other participant also spontaneously decided to switch to the latter variant of the gesture as well.

An interesting result emerged when performing the come gesture. Both participants repeated the come motion repeatedly, although the instructions were that this, like the other gestures, is a single gesture mapped to a single robotic action. None of other gestures was performed repeatedly.

The participants were asked to perform the circling motion with the arm over their heads, and also the revised gesture where the user makes a small circle less conspicuously. The younger participant liked using the former gesture more, while the older liked the latter motion more. The child's overexaggeration of the other gestures explains his preference for the bigger motion, but the adult participant did not cite a reason for his preference.

The participants had a hard time holding the racquet leveled and steady enough for the quadrotor to take off. The child was forced to use both hands to hold the racquet. The adult participant suggested to get rid of the racquet entirely, and takeoff and land on the ground instead.

\section{B. Discussion}

Both participants were very engaged in the gesture-based interaction with the flying robot, and spoke to the quadrotor like a pet while doing the gestures. The child displayed this behavior more than the adult participant. Though at first the younger participant was afraid of the drone, but he later seemed to overcome this fear.

Even though no efforts were taken to give the quadrotor a personality, both participants treated it as if it understood what they said to it, and even showed concern for its wellbeing. The child showed signs of a suspension of disbelief, believing that the quadrotor is a live entity with emotions and intelligent. That said, the quadrotor was viewed as a very expensive toy in the child participant's eyes, and therefore he may be concerned with it getting broken simply because he believed he may get scolded if he caused the damage.

We have not considered using both arms to perform one gesture, and based on the results this seem to be a good expressive method to tell the drone to perform the current task faster, or more powerfully. Similarly, the participants sometimes used the same gesture multiple times in hopes that the quadrotor would come to them faster, or make the gesture 
register when they perceived that the quadrotor might not have seen the action.

From the way both participants unconsciously switch from the original raising and lowering gestures to moving the arm up and down without angling it suggests that the latter gesture is more natural. We will revise the gesture so that the user moves her arm vertically up/down without the angle.

The participants had mixed views on the over-the-head circling motion and the less exaggerated upper arm movement. Thus we are still deliberating which one would be better for controlling the quadrotor circling task.

There seems to be many problems with the use of the racquet, and none of the participants in either of the design critique liked it much. The racquet was too cumbersome, heavy, and difficult to hold steady and leveled for the quadrotor to take off. The child participant was wary of having the quadrotor so close to him, so the takeoff pad did not solve that problem. Therefore, in future iterations, we plan to attempt to abandon the racquet and have the quadrotor takeoff and land on the ground.

\section{FUTURE WORK}

Our main future technical challenge is implementing our gesture based interactions in a real world setting. As a first step towards this goal we plan to utilize the Microsoft's Kinect to recognize the various gestures the user makes.

We are contemplating the possibility of a comparative study comparing flying a collocated drone using gestures, with the experience of flying it with a controller to confirm or disprove that our gestures based method is easier, and that it positively influences the social aspects of the interaction, as we currently believe. Using voice commands and testing more robust gestures mechanisms are also natural extensions of the current work. We would also like to move onto developing a collocated emotional flying robot that communicates with the user using sounds and music.

\section{CONCLUSION}

We attempted to move away from a controller/joystickbased interaction with flying robots in collocated space by using body gestures inspired by falconeering. Using gestures to communicate with a quadrotor when it is nearby is more natural than looking away from the robot and focusing on the controller. We also believe that using gestures when interacting with a collocated flying robot will positively impact the social aspects of the interaction.

From the preliminary design critique sessions we performed with our current prototype, we learned that the body gestures used to control a flying robot is relatively easy to understand and perform. The gestures we proposed were generally well received by the participants in the design critique, with minor adjustments and slight redefinition of the tasks, though the racquet we used for landing and takeoff pad was proven ineffective. We were also glad to learn that as our participants interacted with the quadrotor using the gestures, they became attached to the drone on what can be argued as a social and emotional layer of interaction, and often treated it as if it was a living pet.

Because robots are becoming a part of the common household, it is important to have an easy and natural interaction with them. Our paper presents a preliminary exploration of a novel approach to interacting with flying robots in collocated space, focusing on a more natural and intuitive gesture-based technique in order to communicate with the drones.

\section{REFERENCES}

[1] Ash L. 2009. The modern apprentice. Retrieved Oct. 20, 2010 from http://www.themodernapprentice.com/.

[2] Bainbridge W.A., Hart J., Kim E.S., Scassellati B. The effect of presence on human-robot interaction. In Proc.of the 17th IEEE: Robot and Human Interactive Communications. Munich, Germany, 2008, $701-706$.

[3] Cheng G., Sharlin E. Exploring the use of tangible user interfaces for human-robot interaction: a comparative study. In Proc. of CHI '08: Human-Robot Interaction. Florence, Italy, 2008, 121 - 130.

[4] Croft J. Semaphore Flag signalling System. Retrieved Jan 5, 2011 from http://www.anbg.gov.au/flags/semaphore.html.

[5] Dahlbäck N., Jönsson A., Ahrenberg L. Wizard of Oz studies - why and how. In Intelligent User Interfaces '93. 1993, 193 - 200.

[6] Drury J.L., Riek L., Rackliffe N. A decomposition of UAV-related situation awareness. In Proc. of HRI '06. ACM New York, 2006.

[7] Goodrich M.A., Morse B.S., Gerhardt D., Cooper J.L., Quigley M., Adams J.A., Humphrey C. Supporting wilderness search and rescue using a camera-equipped mini UAV. Journal of Field Robotics Vol. 25 Issue 1-2, 89-110.

[8] Green A., Hüttenrauch H., Topp E.A. Measuring up as an intelligent robot - on the use of high fidelity simulations for human-robot interaction research. In Proc. of the Workshop on Performance Metrics for Intelligent Systems, 2006.

[9] Iba S., Weghe J.M.V., Paredis C.J.J., Khosla, P.K. An architecture for gesture-based control of mobile robots. In Proc. of IEEE Intelligent Robots and Systems. South Korea, 1999, 851-857 Vol. 2.

[10] Landing Signal Officer's standardized signals. Retrieved Jan 5, 2011 from http://upload.wikimedia.org/wikipedia/commons/1/19/LSO_signals _day_US_Navy_1945.jpg.

[11] Quigley M., Goodrich M.A., Randal W.B. Semi-autonomous humanUAV interfaces for fixed-wing mini-UAVs. In Proc of IEEE '04, Intelligent Robots and Systems, Sendai, Japan, 2005, 2457-2462 vol. 3.

[12] Richer J., Drury J. A video game-based framework for analyzing human-robot interaction: characterizing interface design in real-time interactive multimedia applications. In Proc. of ACM SIGCHI/SIGART conference on Human-robot interaction. Salt Lake City, Utah, 2006, 266-273.

[13] Rogalla O., Ehrenmann M., Zöllner R., Becher R., Dillmann R. Using gesture and speech control for commanding a robot assistant. In Proc. of IEEE International Workshop on Robot and Human Interactive Communication, Berlin, 2002, 454-459. 